Communications in Physics, Vol.27, No. 1 (2017), pp. 23-35

DOI:10.15625/0868-3166/27/1/9240

\title{
Invited Paper
}

\section{CHIRALITY OF LIGHT IN HYBRID MODES OF VACUUM-CLAD ULTRATHIN OPTICAL FIBERS}

\author{
FAM LE KIEN ${ }^{1, \dagger}$, TH. BUSCH ${ }^{2}$, VIET GIANG TRUONG ${ }^{3}$ and SÍLE NIC CHORMAIC ${ }^{3,4}$ \\ ${ }^{1}$ Quantum Systems Unit, Okinawa Institute of Science and Technology Graduate University, \\ Onna, Okinawa 904-0495, Japan \\ ${ }^{2}$ Quantum Systems Unit, Okinawa Institute of Science and Technology Graduate University, \\ Onna, Okinawa 904-0495, Japan \\ ${ }^{3}$ Light-Matter Interactions Unit, Okinawa Institute of Science and Technology Graduate \\ University, Onna, Okinawa 904-0495, Japan \\ ${ }^{4}$ School of Chemistry and Physics, University of KwaZulu-Natal, Durban, KwaZulu-Natal, 4001, \\ South Africa \\ ${ }^{\dagger}$ E-mail: kien_pham@hotmail.com
}

Received 21 February 2017

Accepted for publication 9 March 2017

\begin{abstract}
We investigate chirality of light in the quasicircularly polarized fundamental and higherorder hybrid modes of vacuum-clad ultrathin optical fibers. We show that, for a given fiber with the parameters in the range of experimental interest, the higher-order modes have smaller optical chirality per unit energy than the fundamental mode. The sign of the chirality per unit energy of a HE or EH mode is the same as or opposite to, respectively, the sign of the phase circulation direction. When the fiber radius is large enough, the field in a quasicircularly polarized hybrid mode can be superchiral outside the fiber. In particular, outside a fiber with a radius of $600 \mathrm{~nm}$, the fields in the quasicircularly polarized $H E_{11}, H E_{21}$, and $H E_{31}$ modes are superchiral.
\end{abstract}

Keywords: chirality, higher orders, hybrid modes, ultrathin optical fibers.

Classification numbers: 42.81.Qb, 42.25.Ja, 42.50.Tx .

\section{INTRODUCTION}

Guided fields of vacuum-clad ultrathin optical fibers [1-3] penetrate deeply into the surrounding medium and appear as an evanescent wave carrying a significant fraction of the power (C)2017 Vietnam Academy of Science and Technology 
and having a complex polarization pattern [4-6]. Vacuum-clad ultrathin optical fibers have a wide range of potential practical applications [7,8]. They have been used for trapping atoms [9-11], for probing atoms [12-19], molecules [20], quantum dots [21], and color centers in nanodiamonds [22,23], and for mechanical manipulation of small particles [24-28].

An interesting property of guided fields of vacuum-clad ultrathin optical fibers is that they are, in general, chiral. The chirality of an object means that this object cannot be superimposed on its mirror image. The interaction between a chiral light field with a chiral emitter can lead to directional effects [29]. In particular, spontaneous emission and scattering from an atom with a circular dipole near a nanofiber can be asymmetric with respect to the opposite axial propagation directions [30-34]. These directional effects are optical chiral effects and the signature of spin-orbit coupling of light [35-41]. They are due to the existence of a nonzero longitudinal component of the nanofiber-guided field, which oscillates in phase quadrature with respect to the radial transverse component.

Circularly polarized light is the simplest example of chiral light. The chirality of photons in circularly polarized light originates from their spin. It is well known that the spin of photons in circularly polarized light is maximum. This led to a common belief that circularly polarized light must have the maximum chirality. However, this belief is wrong. Although chirality and spin are related to each other, they are different characteristics of light. Tang and Cohen have introduced a chirality measure for the interaction between chiral light and chiral molecules with mixed electricmagnetic dipole polarizability. They have shown that the local values of this measure of chirality at the nodes of a circularly polarized standing wave can be larger than for circularly polarized light $[42,43]$. They termed such light as being superchiral. A disadvantage of the superchiral light construction scheme of Refs. $[42,43]$ is that the superchirality appears at the nodes of a standing wave, where the field intensity and hence the molecular excitation rate are small.

The purpose of this work is to study chirality of light in hybrid modes of vacuum-clad ultrathin optical fibers. We calculate, analytically and numerically, the chirality measure introduced by Tang and Cohen $[42,43]$.

The paper is organized as follows. In Sec. II we briefly review the basic properties of hybrid modes of optical fibers. In Sec. III we calculate the chirality of guided light and our conclusions are given in Sec. IV.

\section{HYBRID MODES OF OPTICAL FIBERS}

In this section, we review the basic properties of hybrid modes of optical fibers.

Consider the model of a step-index fiber that is a dielectric cylinder of radius $a$ and refractive index $n_{1}$, surrounded by an infinite background medium of refractive index $n_{2}$, where $n_{2}<n_{1}$. We use Cartesian coordinates $\{x, y, z\}$, where $z$ is the coordinate along the fiber axis. We also use cylindrical coordinates $\{r, \varphi, z\}$, where $r$ and $\varphi$ are the polar coordinates in the fiber transverse plane $x y$.

For a guided light field of frequency $\omega$ (free-space wavelength $\lambda=2 \pi c / \omega$ and free-space wave number $k=\omega / c$ ), the propagation constant $\beta$ is determined by the fiber eigenvalue equation [44]. This eigenvalue equation leads to hybrid HE and EH modes, and to TE and TM modes [44]. We do not consider TE and TM modes in this paper because the integral chirality of light in these modes and the corresponding chirality density are zero. 
Hybrid $\mathrm{HE}$ and $\mathrm{EH}$ modes are labeled as $\mathrm{HE}_{l m}$ and $\mathrm{EH}_{l m}$, where $l=1,2, \ldots$ and $m=1,2, \ldots$ are the azimuthal and radial mode orders, respectively. The azimuthal mode order $l$ determines the helical phasefront and the associated phase gradient in the fiber transverse plane. The radial mode order $m$ implies that the $\mathrm{HE}_{l m}$ or $\mathrm{EH}_{l m}$ mode is the $m$-th solution to the corresponding eigenvalue equation.

We examine the mode functions of hybrid modes [44]. We present the electric and magnetic components of the field in the form $\mathbf{E}=\left(\mathscr{E} e^{-i \omega t}+\right.$ c.c. $) / 2$ and $\mathbf{H}=\left(\mathscr{H} e^{-i \omega t}+\right.$ c.c. $) / 2$, where $\mathscr{E}$ and $\mathscr{H}$ are the envelopes. The spatial functions $\mathscr{E}$ and $\mathscr{H}$ obey the Helmholtz equation and are called the mode functions. For a guided mode with a propagation constant $\beta$ and an azimuthal mode order $l$, we can write $\mathscr{E}=\mathbf{e} e^{i \beta z+i l \varphi}$ and $\mathscr{H}=\mathbf{h} e^{i \beta z+i l \varphi}$. Here, $\mathbf{e}$ and $\mathbf{h}$ are the mode profile functions of the electric and magnetic components of the field, and $\beta$ and $l$ can have positive and negative values. The explicit expressions for the mode profile functions $\mathbf{e}$ and $\mathbf{h}$ can be found in Refs. [44,45].

For convenience, we use the notations $\beta>0$ for the propagation constant and $l>0$ for the azimuthal mode order. We introduce the index $f=+1$ or -1 (or simply $f=+$ or - ) for the positive or negative propagation direction, which indicates that the propagation phase factor is $e^{i \beta z}$ or $e^{-i \beta z}$. We also introduce the index $p=+1$ or -1 (or simply $p=+$ or - ) for the counterclockwise or clockwise phase circulation direction, which indicates that the azimuthal phase factor is $e^{i l \varphi}$ or $e^{-i l \varphi}$. We label quasicircularly polarized hybrid modes by the mode index $\mu=(f l p)$.

In cylindrical coordinates, the mode profile functions $\mathbf{e}^{(f l p)}(\mathbf{r})$ and $\mathbf{h}^{(f l p)}(\mathbf{r})$ of the electric and magnetic components of quasicircularly polarized hybrid modes are given by

$$
\begin{aligned}
\mathbf{e}^{(f l p)} & =\hat{\mathbf{r}} e_{r}+p \hat{\varphi} e_{\varphi}+f \hat{\mathbf{z}} e_{z}, \\
\mathbf{h}^{(f l p)} & =f p \hat{\mathbf{r}} h_{r}+f \hat{\varphi} h_{\varphi}+p \hat{\mathbf{z}} h_{z} .
\end{aligned}
$$

Here, the notations $\hat{\mathbf{r}}=\hat{\mathbf{x}} \cos \varphi+\hat{\mathbf{y}} \sin \varphi, \hat{\varphi}=-\hat{\mathbf{x}} \sin \varphi+\hat{\mathbf{y}} \cos \varphi$, and $\hat{\mathbf{z}}$ stand for the unit basis vectors of the cylindrical coordinate system $\{r, \varphi, z\}$, with $\hat{\mathbf{x}}$ and $\hat{\mathbf{y}}$ being the unit basis vectors of the Cartesian coordinate system for the fiber transverse plane $x y$. The notations $e_{r}, e_{\varphi}$, and $e_{z}$ and the notations $h_{r}, h_{\varphi}$, and $h_{z}$ stand for the cylindrical-coordinate components of the electric and magnetic mode functions $\mathbf{e}$ and $\mathbf{h}$ for the corresponding hybrid mode with the positive propagation direction $f=+$ and the counterclockwise phase circulation direction $p=+$. Note that $\mathbf{e}$ and $\mathbf{h}$ depend on the type (HE or EH) of the mode and on the mode orders $l$ and $m$.

An important property of the mode functions is that the longitudinal components $e_{z}$ and $h_{z}$ are nonvanishing and are in quadrature ( $\pi / 2$ out of phase) with the radial components $e_{r}$ and $h_{r}$, respectively. In addition, the azimuthal components $e_{\varphi}$ and $h_{\varphi}$ are also nonvanishing and are in quadrature with the radial components $e_{r}$ and $h_{r}$, respectively. Note that the full mode functions for quasicircularly polarized hybrid modes are given by

$$
\begin{aligned}
\mathscr{E}^{(f l p)} & =\mathbf{e}^{(f l p)} e^{i f \beta z+i p l \varphi}, \\
\mathscr{H}^{(f l p)} & =\mathbf{h}^{(f l p)} e^{i f \beta z+i p l \varphi} .
\end{aligned}
$$

We are interested in ultrathin fibers, whose diameters are on the order of a micron. Such fibers can support not only the fundamental $\mathrm{HE}_{11}$ mode but also several higher-order modes in the optical region. We note that the excitation of higher-order modes has been studied $[46,47]$. The production of ultrathin fibers for higher-order mode propagation with high transmission has been 
demonstrated [48-50]. Experimental studies on the interaction between higher-order modes and atoms [19] or particles [27,28] have been reported.

\section{CHIRALITY}

According to [42], the cycle-averaged optical chirality density of a monochromatic field can be characterized by the quantity

$$
\rho^{\text {chir }}=\frac{c \varepsilon_{0} n^{2}}{4 \omega} \operatorname{Re}\left[\mathscr{E}^{*} \cdot(\nabla \times \mathscr{E})\right]+\frac{c \mu_{0}}{4 \omega} \operatorname{Re}\left[\mathscr{H}^{*} \cdot(\nabla \times \mathscr{H})\right] .
$$

Here, $n(r)=n_{1}$ for $r<a$ and $n_{2}$ for $r>a$. The quantity $\rho^{\text {chir }}$ has been studied in Refs. [42,43, 51-54]. When we use the Maxwell equations $\nabla \times \mathscr{E}=i \omega \mu_{0} \mathscr{H}$ and $\nabla \times \mathscr{H}=-i \omega \varepsilon_{0} n^{2} \mathscr{E}$ for the fields in the frequency domain, we obtain [53]

$$
\rho^{\mathrm{chir}}=\frac{n^{2}}{2 c} \operatorname{Im}\left[\mathscr{E} \cdot \mathscr{H}^{*}\right]
$$

For TE and TM guided modes, the chirality density (4) is zero.

The optical chirality density defined by Eq. (4) can be measured from the asymmetry in the rates of excitation between a small chiral molecule and its mirror image [42,43]. According to Ref. [55], there is no single measure of chirality and, for any measure, there exists a chiral body for which the measure is zero.

The chirality of a light beam is closely related to the optical helicity. Indeed, the cycleaveraged optical helicity density of a monochromatic field is given by [51,56-60]

$$
\rho^{\text {hlcy }}=\frac{1}{4 c} \operatorname{Re}\left(\mathscr{A} \cdot \mathscr{H}^{*}\right)-\frac{\varepsilon_{0} n^{2}}{4} \operatorname{Re}\left(\mathscr{C} \cdot \mathscr{E}^{*}\right),
$$

where $\mathscr{A}$ and $\mathscr{C}$ are the positive frequency components of the magnetic and electric vector potentials, respectively. With the help of the relations $\mathscr{A}=\mathscr{E} / i \omega$ and $n^{2} \mathscr{C}=\mu_{0} c \mathscr{H} / i \omega$ between the vector potentials and the fields in the frequency domain, we obtain [51,56-60]

$$
\rho^{\text {hlcy }}=\frac{1}{2 c \omega} \operatorname{Im}\left(\mathscr{E} \cdot \mathscr{H}^{*}\right) .
$$

It is clear that $\rho^{\text {chir }}=n^{2} \omega \rho^{\text {hlcy }}$. Thus, in the frequency domain, the chirality density is proportional to the helicity density. The proportionality factor is $n^{2} \omega$.

For quasicircularly polarized hybrid modes, we find from Eq. (4) the following expression for the chirality density:

$$
\rho^{\mathrm{chir}}=f p \frac{n^{2}}{2 c} \operatorname{Im}\left(e_{r} h_{r}^{*}+e_{\varphi} h_{\varphi}^{*}+e_{z} h_{z}^{*}\right) .
$$

For guided light, the optical chirality per unit length is

$$
J^{\text {chir }}=\int \rho^{\text {chir }} d \mathbf{r}
$$

where $\int d \mathbf{r}=\int_{0}^{2 \pi} d \varphi \int_{0}^{\infty} r d r$. It is clear from Eqs. (7) and (8) that the sign of chirality per unit length changes when the propagation direction $f$ or the phase circulation direction $p$ is reversed. 
We calculate analytically the chirality per unit length (8). For this purpose, we use the explicit expressions for the mode functions $\mathbf{e}$ and $\mathbf{h}[44,45]$. When we insert these expressions into Eq. (7) and calculate the integral (8), we obtain the explicit expression

$$
\begin{aligned}
J^{\text {chir }}= & f p|A|^{2} \frac{\pi a^{2} \varepsilon_{0} n_{1}^{2} \beta}{4 h^{2} k}\left\{n_{1}^{2} k^{2}(1-s)\left(1-s_{1}\right)\left[J_{l-1}^{2}(h a)-J_{l-2}(h a) J_{l}(h a)\right]\right. \\
& -n_{1}^{2} k^{2}(1+s)\left(1+s_{1}\right)\left[J_{l+1}^{2}(h a)-J_{l+2}(h a) J_{l}(h a)\right] \\
& \left.-2 h^{2} s\left[J_{l}^{2}(h a)-J_{l+1}(h a) J_{l-1}(h a)\right]\right\} \\
& +f p|A|^{2} \frac{\pi a^{2} \varepsilon_{0} n_{2}^{2} \beta}{4 q^{2} k} \frac{J_{l}^{2}(h a)}{K_{l}^{2}(q a)}\left\{n_{2}^{2} k^{2}(1-s)\left(1-s_{2}\right)\left[K_{l-2}(q a) K_{l}(q a)-K_{l-1}^{2}(q a)\right]\right. \\
& -n_{2}^{2} k^{2}(1+s)\left(1+s_{2}\right)\left[K_{l+2}(q a) K_{l}(q a)-K_{l+1}^{2}(q a)\right] \\
& \left.-2 q^{2} s\left[K_{l+1}(q a) K_{l-1}(q a)-K_{l}^{2}(q a)\right]\right\} .
\end{aligned}
$$

Here, the parameters $h=\left(n_{1}^{2} k^{2}-\beta^{2}\right)^{1 / 2}$ and $q=\left(\beta^{2}-n_{2}^{2} k^{2}\right)^{1 / 2}$ characterize the scales of the spatial variations of the field inside and outside the fiber, respectively. The parameters $s, s_{1}$, and $s_{2}$ are given as

$$
\begin{aligned}
s & =l\left(\frac{1}{h^{2} a^{2}}+\frac{1}{q^{2} a^{2}}\right)\left[\frac{J_{l}^{\prime}(h a)}{h a J_{l}(h a)}+\frac{K_{l}^{\prime}(q a)}{q a K_{l}(q a)}\right]^{-1} \\
s_{1} & =\frac{\beta^{2}}{k^{2} n_{1}^{2}} s, \\
s_{2} & =\frac{\beta^{2}}{k^{2} n_{2}^{2}} s .
\end{aligned}
$$

The coefficient $A$ is a constant that can be determined from the propagating power of the field.

The optical chirality per unit length can be normalized to the energy per unit length, which is given by

$$
U=\frac{\varepsilon_{0}}{4} \int n^{2}|\mathscr{E}|^{2} d \mathbf{r}+\frac{\mu_{0}}{4} \int|\mathscr{H}|^{2} d \mathbf{r} .
$$

The first and second terms on the right-hand side of expression (11) correspond to the electric and magnetic parts of the energy of the field, respectively. For guided modes, these parts are equal to each other. We have $U=U_{\text {in }}+U_{\text {out }}$, where $U_{\text {in }}$ and $U_{\text {out }}$ are the energies per unit length inside and outside the fiber and are given as $[44,45]$

$$
\begin{aligned}
U_{\text {in }}= & |A|^{2} \frac{\pi a^{2} \varepsilon_{0} n_{1}^{2}}{4}\left\{\frac{1}{2 h^{2}}\left[\beta^{2}(1-s)^{2}+n_{1}^{2} k^{2}\left(1-s_{1}\right)^{2}\right]\left[J_{l-1}^{2}(h a)-J_{l-2}(h a) J_{l}(h a)\right]\right. \\
& +\frac{1}{2 h^{2}}\left[\beta^{2}(1+s)^{2}+n_{1}^{2} k^{2}\left(1+s_{1}\right)^{2}\right]\left[J_{l+1}^{2}(h a)-J_{l+2}(h a) J_{l}(h a)\right] \\
& \left.+\left(1+\frac{\beta^{2} s^{2}}{n_{1}^{2} k^{2}}\right)\left[J_{l}^{2}(h a)-J_{l-1}(h a) J_{l+1}(h a)\right]\right\}
\end{aligned}
$$


and

$$
\begin{aligned}
U_{\text {out }}= & |A|^{2} \frac{\pi a^{2} \varepsilon_{0} n_{2}^{2}}{4} \frac{J_{l}^{2}(h a)}{K_{l}^{2}(q a)}\left\{\frac{1}{2 q^{2}}\left[\beta^{2}(1-s)^{2}+n_{2}^{2} k^{2}\left(1-s_{2}\right)^{2}\right]\left[K_{l-2}(q a) K_{l}(q a)-K_{l-1}^{2}(q a)\right]\right. \\
& +\frac{1}{2 q^{2}}\left[\beta^{2}(1+s)^{2}+n_{2}^{2} k^{2}\left(1+s_{2}\right)^{2}\right]\left[K_{l+2}(q a) K_{l}(q a)-K_{l+1}^{2}(q a)\right] \\
& \left.+\left(1+\frac{\beta^{2} s^{2}}{n_{2}^{2} k^{2}}\right)\left[K_{l-1}(q a) K_{l+1}(q a)-K_{l}^{2}(q a)\right]\right\} .
\end{aligned}
$$

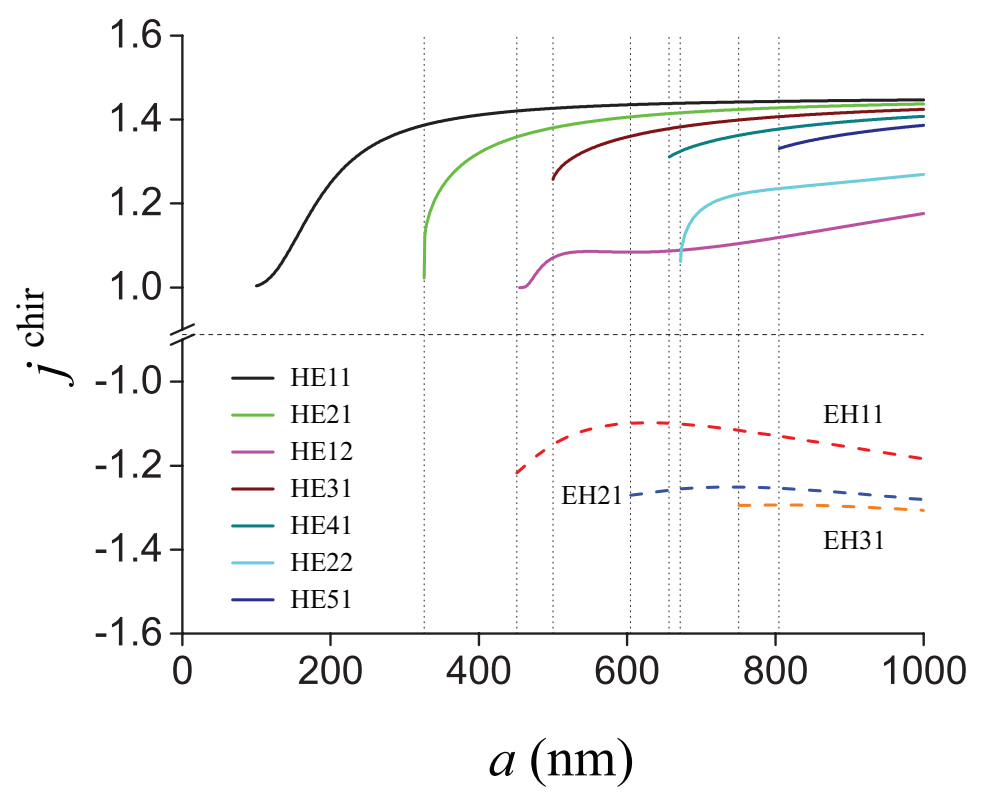

Fig. 1. (Color online) Chirality per unit energy $j^{\text {chir }}$ as a function of the fiber radius $a$ for quasicircularly polarized hybrid modes with the positive propagation direction $f=+$ and the positive phase circulation direction $p=+$. The refractive indices of the fiber and the surrounding medium are $n_{1}=1.4537$ and $n_{2}=1$, respectively. The wavelength of light is $\lambda=780 \mathrm{~nm}$. The vertical dotted lines indicate the positions of the cutoffs for higher-order modes. The horizontal dotted line separates the positive and negative sides of the vertical axis.

We introduce the notation $j^{\text {chir }}=J^{\text {chir }} / U$ for the chirality per unit energy of guided light. We show $j^{\text {chir }}$ as a function of the fiber radius $a$ in Fig. 1. We see from the figure that, for the positive propagation direction $f=+$ and the positive phase circulation direction $p=+$, the chirality per unit energy $j^{\text {chir }}$ is positive for HE and negative for EH modes. We note that the chirality per unit energy of a circularly polarized light field in a dielectric medium of refractive index $n$ is equal to $n$. We observe that the absolute values of $j^{\text {chir }}$ obtained for the guided modes do not exceed the refractive index $n_{1}$ of the fiber. 


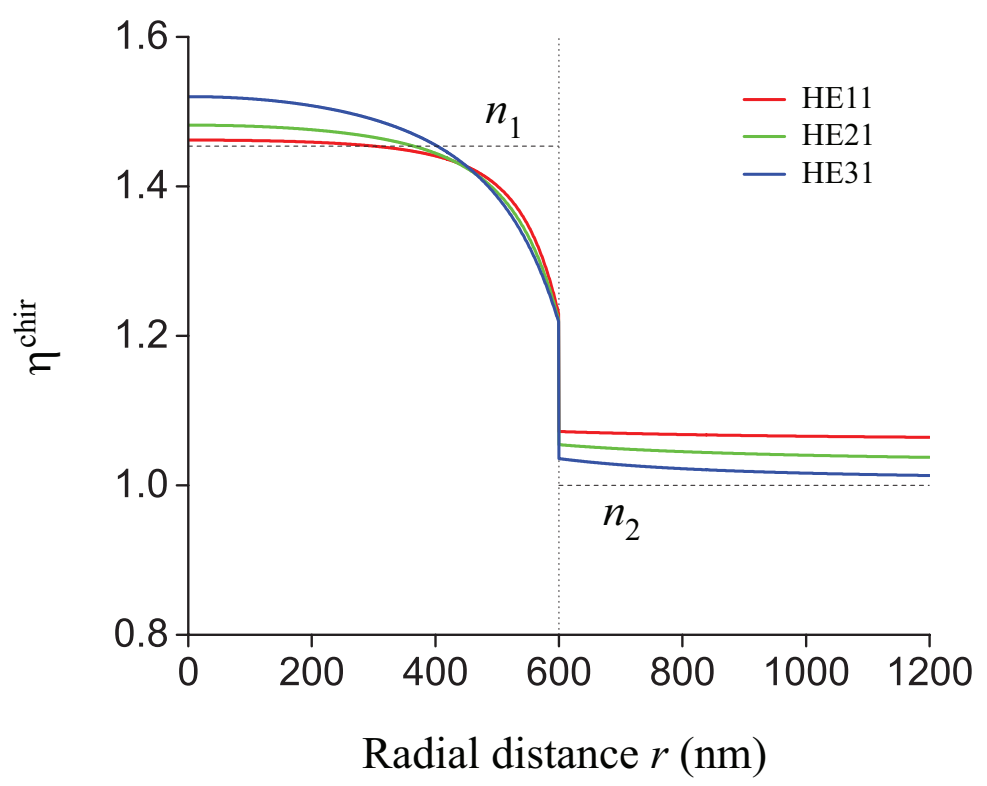

Fig. 2. Chirality factor $\eta^{\text {chir }}$ as a function of the radial distance $r$ for the counterclockwise quasicircularly polarized $\mathrm{HE}_{11}$ (red line), $\mathrm{HE}_{21}$ (green line), and $\mathrm{HE}_{31}$ (blue line) modes. The fiber radius is $a=600 \mathrm{~nm}$. Other parameters are as for Fig. 1. The vertical dotted line indicates the position of the fiber surface. The horizontal dotted lines indicate the refractive index values $n_{1}=1.4537$ and $n_{2}=1$.

According to $[42,43]$, the asymmetry in the excitation rates between a small chiral molecule and its mirror image is proportional to the factor

$$
\eta^{\text {chir }}=\frac{\rho^{\text {chir }}}{2 u_{e}}
$$

where

$$
u_{e}=\frac{\varepsilon_{0} n^{2}}{4}|\mathscr{E}|^{2}=\frac{\varepsilon_{0} n^{2}}{4}\left(\left|e_{r}\right|^{2}+\left|e_{\varphi}\right|^{2}+\left|e_{z}\right|^{2}\right)
$$

is the local electric energy density of the field. Note that the value of the factor $\eta^{\text {chir }}$ for a counterclockwise or clockwise circularly polarized field in a medium with refractive index $n$ is equal to $n$ or $-n$. According to [42,43], a light field for which the absolute value of the chirality factor $\eta^{\text {chir }}$ is larger than the refractive index $n$ in a spatial region is called a superchiral field.

We plot in Fig. 2 the factor $\eta^{\text {chir }}$ as a function of the radial distance $r$ for the quasicircularly polarized $\mathrm{HE}_{11}, \mathrm{HE}_{21}$, and $\mathrm{HE}_{31}$ modes. We observe from the figure that the values of $\eta^{\text {chir }}$ inside and outside the fiber are very different from each other. We see that $\eta^{\text {chir }}$ is discontinuous at the position of the fiber surface. This discontinuity results from the boundary condition for the normal (radial) component $e_{r}$ of the electric field. Since the difference between the refractive indices $n_{1}$ of the silica core and $n_{2}$ of the vacuum cladding is large, the discontinuity of $\eta^{\text {chir }}$ at the fiber surface is dramatic. We observe from the figure that $\eta^{\text {chir }}$ is positive. Comparison between the colored curves of Fig. 2 shows that, outside the fiber, $\eta^{\text {chir }}$ is larger for the $\mathrm{HE}_{11}$ mode (red curve) than for 
the higher-order $\mathrm{HE}_{21}$ (green curve) and $\mathrm{HE}_{31}$ (blue curve) modes. An interesting feature is that, outside the fiber, the values of $\eta^{\text {chir }}$ for the $\mathrm{HE}_{11}, \mathrm{HE}_{21}$, and $\mathrm{HE}_{31}$ modes are larger than unity. Although the enhancement of $\eta^{\text {chir }}$ is significant, it is limited by the magnitude of the fiber radius and the difference between the refractive indices of the fiber and the surrounding medium.

Thus, for an ultrathin fiber with a radius of $600 \mathrm{~nm}$, superchirality of light can be obtained for the quasicircularly polarized $\mathrm{HE}_{11}, \mathrm{HE}_{21}$, and $\mathrm{HE}_{31}$ modes. Such superchirality appears as a consequence of the complex polarization structure of the guided field. The total internal reflection of light from the fiber surface is the physical mechanism that leads to this superchirality. This mechanism is different from the interference between two counterpropagating circularly polarized plane waves with opposite handedness, equal frequency, and slightly different intensity, which was used by Tang and Cohen to produce a standing-wave superchiral light field $[42,43]$. An advantage of the waveguide scheme is that superchirality of the $\mathrm{HE}_{11}, \mathrm{HE}_{21}$, and $\mathrm{HE}_{31}$ modes can occur everywhere in the evanescent region of the field (outside the fiber). In contrast, superchirality of the field constructed in the standing-wave scheme is limited to the nodes, where the intensity is small. One can increase the enhancement of the chirality factor in the waveguide scheme by increasing the fiber radius and by using a material with a higher refractive index.

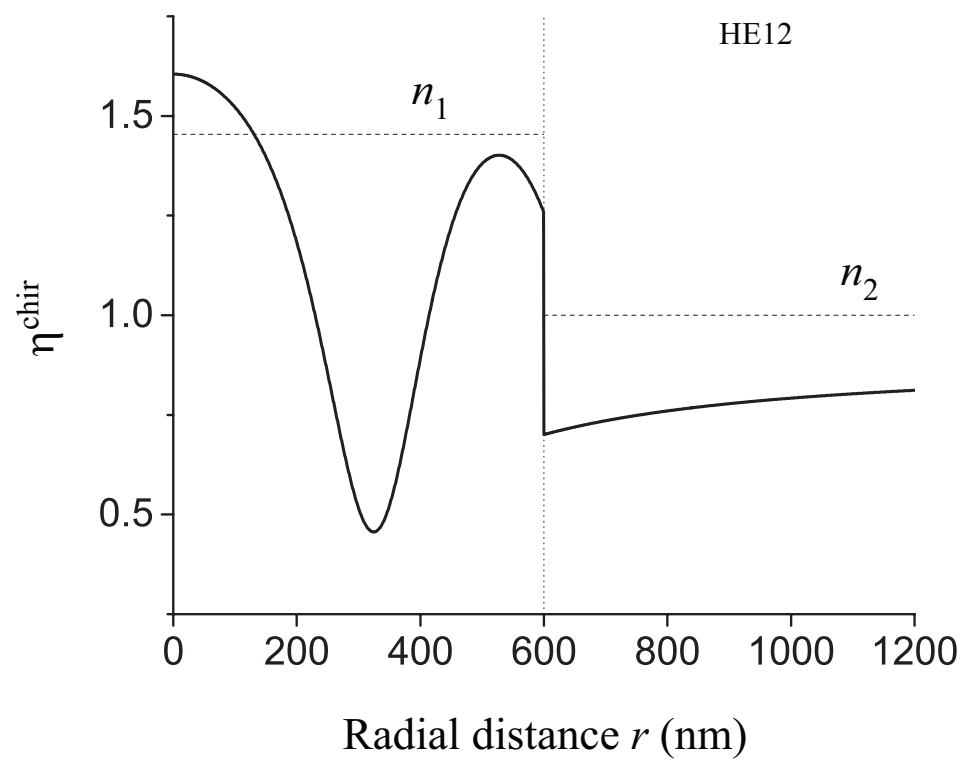

Fig. 3. Chirality factor $\eta^{\text {chir }}$ as a function of the radial distance $r$ for the counterclockwise quasicircularly polarized higher-order $\mathrm{HE}_{12}$ mode. The parameters used are the same as for Fig. 2. The vertical dotted line indicates the position of the fiber surface. The horizontal dotted lines indicate the refractive index values $n_{1}=1.4537$ and $n_{2}=1$.

In Fig. 3, we plot $\eta^{\text {chir }}$ as a function of $r$ for the quasicircularly polarized $\mathrm{HE}_{12}$ mode. We see from the figure that $\eta^{\text {chir }}$ is positive. Outside the fiber, this factor is smaller than unity.

In Fig. 4, we plot $\eta^{\text {chir }}$ as a function of $r$ for the quasicircularly polarized $\mathrm{EH}_{11}$ mode. We see from the figure that $\eta^{\text {chir }}$ is positive in a limited region containing the fiber center, but is negative everywhere else. This behavior is consistent with the fact that the chirality per unit energy 


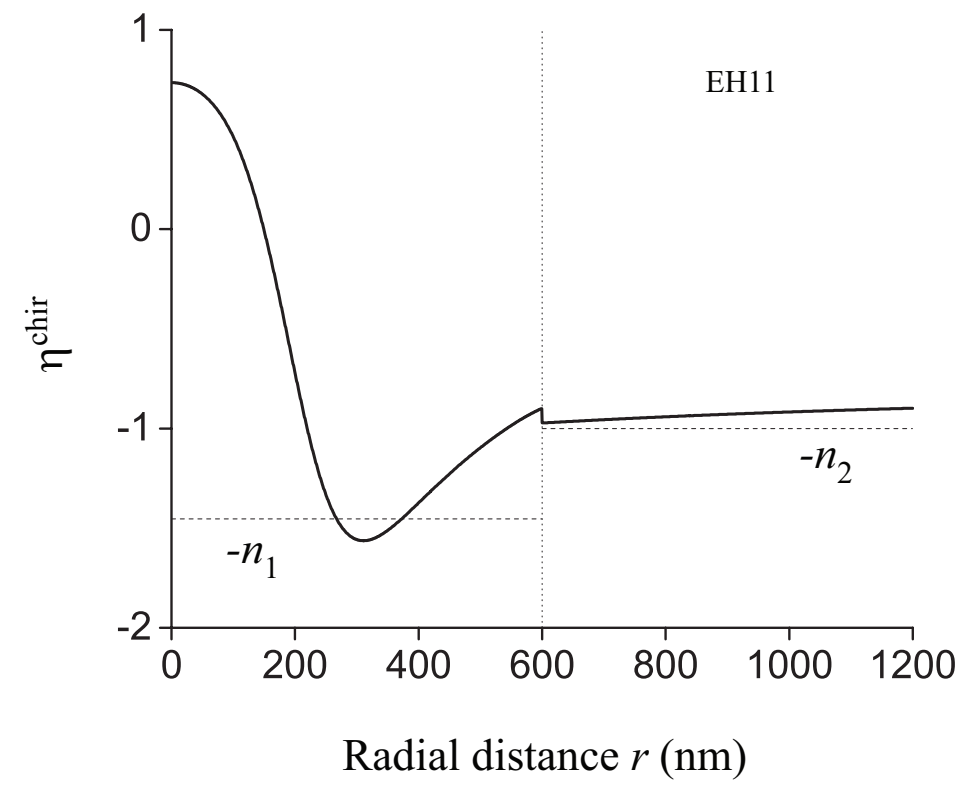

Fig. 4. Chirality factor $\eta^{\text {chir }}$ as a function of the radial distance $r$ for the counterclockwise quasicircularly polarized higher-order $\mathrm{EH}_{11}$ mode. The parameters used are the same as for Fig. 2. The vertical dotted line indicates the position of the fiber surface. The horizontal dotted lines indicate the values $-n_{1}=-1.4537$ and $-n_{2}=-1$.

$j^{\text {chir }}$ is negative for EH modes (see Fig. 1). Outside the fiber, $\eta^{\text {chir }}$ is negative, and its absolute value is smaller than unity.

Thus, according to Figs. 3 and 4, for a fiber with a radius of $600 \mathrm{~nm}$, the fields in the quasicircularly polarized $\mathrm{HE}_{12}$ and $\mathrm{EH}_{11}$ modes are not superchiral outside the fiber. However, these field are superchiral in some regions inside the fiber. Furthermore, additional calculations show that, when the fiber radius is large enough, the fields in the $\mathrm{HE}_{12}$ and $\mathrm{EH}_{11}$ modes can become superchiral outside the fiber (see also Fig. 5).

We see from Figs. 2-4 that, in the limit of large radial distances, the chirality factor $\eta^{\text {chir }}$ slowly varies and tends to a finite value $\eta_{\infty}^{\text {chir }}$, which depends on the mode type and the azimuthal and radial mode orders. We confirm this by performing a simple analysis below.

In the limit $r \rightarrow \infty$, we have

$$
\begin{aligned}
e_{r} & =i A \frac{\beta}{q} \frac{J_{l}(h a)}{K_{l}(q a)} \sqrt{\frac{\pi}{2 q r}} e^{-q r}, \\
e_{\varphi} & =A \frac{\beta s}{q} \frac{J_{l}(h a)}{K_{l}(q a)} \sqrt{\frac{\pi}{2 q r}} e^{-q r}, \\
e_{z} & =A \frac{J_{l}(h a)}{K_{l}(q a)} \sqrt{\frac{\pi}{2 q r}} e^{-q r},
\end{aligned}
$$


and

$$
\begin{aligned}
h_{r} & =-A \frac{\omega \varepsilon_{0} n_{2}^{2} s_{2}}{q} \frac{J_{l}(h a)}{K_{l}(q a)} \sqrt{\frac{\pi}{2 q r}} e^{-q r}, \\
h_{\varphi} & =i A \frac{\omega \varepsilon_{0} n_{2}^{2}}{q} \frac{J_{l}(h a)}{K_{l}(q a)} \sqrt{\frac{\pi}{2 q r}} e^{-q r}, \\
h_{z} & =i A \frac{\beta s}{\omega \mu_{0}} \frac{J_{l}(h a)}{K_{l}(q a)} \sqrt{\frac{\pi}{2 q r}} e^{-q r} .
\end{aligned}
$$

Then, Eqs. (7) and (15) yield the asymptotic expressions

$$
\rho^{\text {chir }}=-f p|A|^{2} \frac{\varepsilon_{0} n_{2}^{2}}{k q^{2}} \beta^{3} s \frac{J_{l}^{2}(h a)}{K_{l}^{2}(q a)} \frac{\pi}{2 q r} e^{-2 q r}
$$

and

$$
u_{e}=|A|^{2} \frac{\varepsilon_{0} n_{2}^{2}}{4 q^{2}}\left[q^{2}+\beta^{2}\left(1+s^{2}\right)\right] \frac{J_{l}^{2}(h a)}{K_{l}^{2}(q a)} \frac{\pi}{2 q r} e^{-2 q r},
$$

which lead to

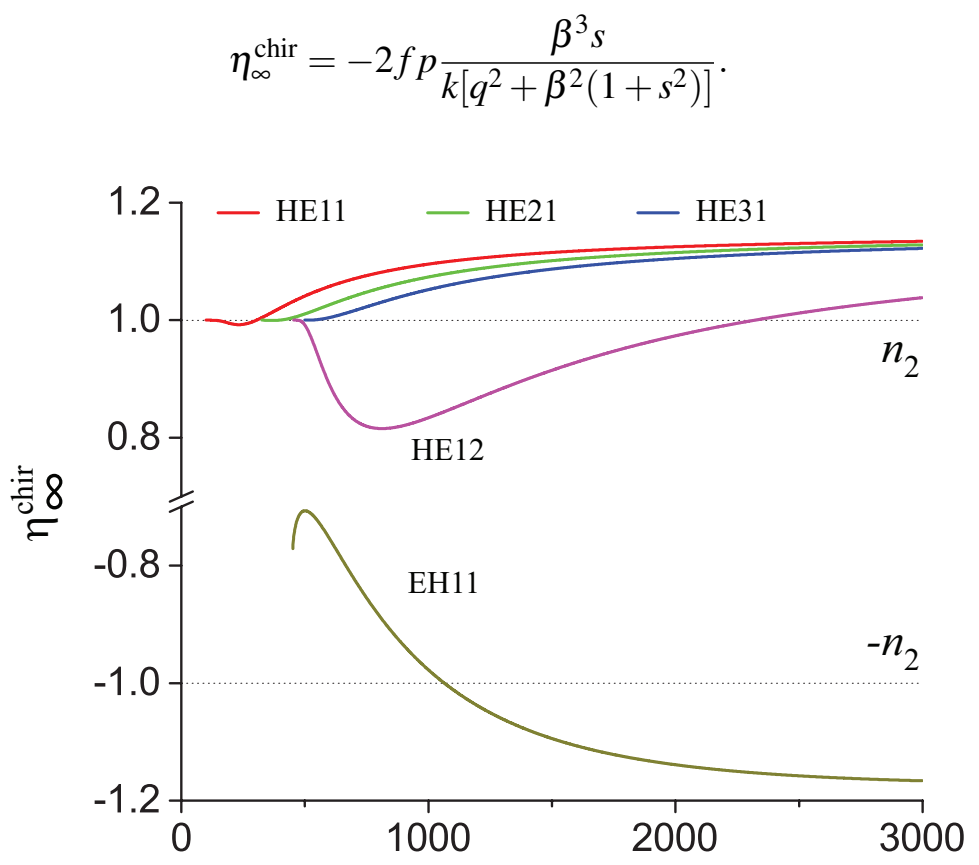

Fiber radius $a(\mathrm{~nm})$

Fig. 5. Dependence of the limiting value $\eta_{\infty}^{\text {chir }}$ of the chirality factor $\eta^{\text {chir }}$ on the fiber radius $a$ for the counterclockwise quasicircularly polarized $\mathrm{HE}_{11}, \mathrm{HE}_{21}, \mathrm{HE}_{31}, \mathrm{HE}_{12}$, and $\mathrm{EH}_{11}$ modes. All other parameters are as for Fig. 1. The horizontal dotted lines indicate the values $n_{2}=1$ and $-n_{2}=-1$. 
The dependence of $\eta_{\infty}^{\text {chir }}$ on the fiber radius $a$ is shown in Fig. 5. Expression (20) and Fig. 5 show clearly that $\eta_{\infty}^{\text {chir }}$ depends on the parameters of the fiber and the mode. One can see from Fig. 5 that, when the fiber radius is large enough, the absolute value of the limiting value $\eta_{\infty}^{\text {chir }}$ is larger than unity, that is, the mode is superchiral outside the fiber. In general, $\eta_{\infty}^{\text {chir }}$ is different from the values $\eta_{\text {circ }}^{\text {chir }}= \pm 1$ for circularly polarized light in free space.

\section{SUMMARY AND DISCUSSION}

We have studied chirality of light in the quasicircularly polarized fundamental and higherorder hybrid modes of vacuum-clad ultrathin optical fibers. We have shown that, for a given fiber with parameters in the range of experimental interest, the higher-order hybrid modes have smaller optical chirality per unit energy than the fundamental mode. In addition, the sign of the chirality per unit energy of a HE or EH mode is the same as or opposite to, respectively, the sign of the phase circulation direction. We have found that, when the fiber radius is large enough, the field in a quasicircularly polarized hybrid mode can be superchiral outside the fiber. In particular, outside a fiber with a radius of $600 \mathrm{~nm}$, the fields in the quasicircularly polarized $\mathrm{HE}_{11}, \mathrm{HE}_{21}$, and $\mathrm{HE}_{31}$ modes are superchiral.

Unlike the standing-wave scheme of Tang and Cohen $[42,43]$, where superchirality is limited to the nodes of a standing wave, the waveguide scheme allows one to produce a propagating guided field with superchirality everywhere in the evanescent region of the field outside a fiber. The large volume with enhanced local chirality is a favorable factor that could be used to excite extended ensembles of chiral molecules, for example. A possible experiment could be realized by sending light into a quasicircularly polarized fundamental or higher-order mode of a microfiber surrounded by a gas, a liquid, or a thin layer of chiral molecules with electric-magnetic dipole polarizability (for example, a biperylene derivative). The excitation rate of the chiral molecules will depend on the handedness of the light field and the molecules. The intensity of fluorescence from excited molecules will change significantly when we reverse the phase circulation direction or the propagation direction of the excitation light, or replace an enantiomer by its opposite enantiomer. The dissymmetry of the rate would be enhanced since the excitation light is superchiral. Our results may open up a new way to sort, select, control, and manipulate chiral molecules and particles.

\section{ACKNOWLEDGMENT}

We acknowledge support for this work from the Okinawa Institute of Science and Technology Graduate University. S.N.C. and T.B. are grateful to JSPS for partial support from a Grant-inAid for Scientific Research (Grant No. 26400422).

\section{REFERENCES}

[1] L. Tong, R. R. Gattass, J. B. Ashcom, S. He, J. Lou, M. Shen, I. Maxwell, and E. Mazur, Nature (London) 426 (2003) 816.

[2] T. A. Birks, W. J. Wadsworth, and P. St. J. Russell, Opt. Lett. 25 (2000) 1415; S. G. Leon-Saval, T. A. Birks, W. J. Wadsworth, P. St. J. Russell, and M. W. Mason, in Conference on Lasers and Electro-Optics (CLEO), Technical Digest, postconference ed. (Optical Society of America, Washington, D.C., 2004), paper CPDA6.

[3] J. C. Knight, G. Cheung, F. Jacques, and T. A. Birks, Opt. Lett. 22 (1997) 1129; M. Cai and K. Vahala, ibid. 26 (2001) 884 . 
[4] J. Bures and R. Ghosh, J. Opt. Soc. Am. A 16 (1999) 1992.

[5] L. Tong, J. Lou, and E. Mazur, Opt. Express 12 (2004) 1025.

[6] Fam Le Kien, J. Q. Liang, K. Hakuta, and V. I. Balykin, Opt. Commun. 242 (2004) 445.

[7] M. J. Morrissey, K. Deasy, M. Frawley, R. Kumar, E. Prel, L. Russell, V. G. Truong, and S. Nic Chormaic, Sensors 13 (2013) 10449.

[8] T. Nieddu, V. Gokhroo, and S. Nic Chormaic, J. Opt. 18 (2016) 053001.

[9] V. I. Balykin, K. Hakuta, Fam Le Kien, J. Q. Liang, and M. Morinaga, Phys. Rev. A 70 (2004) 011401(R); Fam Le Kien, V. I. Balykin, and K. Hakuta, ibid. 70 (2004) 063403.

[10] E. Vetsch, D. Reitz, G. Sagué, R. Schmidt, S. T. Dawkins, and A. Rauschenbeutel, Phys. Rev. Lett. 104 (2010) 203603.

[11] A. Goban, K. S. Choi, D. J. Alton, D. Ding, C. Lacroûte, M. Pototschnig, T. Thiele, N. P. Stern, and H. J. Kimble, Phys. Rev. Lett. 109 (2012) 033603.

[12] P. Domokos, P. Horak, and H. Ritsch, Phys. Rev. A 65 (2002) 033832.

[13] Fam Le Kien, V. I. Balykin, and K. Hakuta, Phys. Rev. A 73 (2006) 013819.

[14] K. P. Nayak, P. N. Melentiev, M. Morinaga, Fam Le Kien, V. I. Balykin, and K. Hakuta, Opt. Express 15 (2007) 5431.

[15] K. P. Nayak, Fam Le Kien, M. Morinaga, and K. Hakuta, Phys. Rev. A 79 (2009) 021801(R).

[16] S. T. Dawkins, R. Mitsch, D. Reitz, E. Vetsch, and A. Rauschenbeutel, Phys. Rev. Lett. 107 (2011) 243601.

[17] D. Reitz, C. Sayrin, R. Mitsch, P. Schneeweiss, and A. Rauschenbeutel, Phys. Rev. Lett. 110 (2013) 243603.

[18] L. Russell, R. Kumar, V. B. Tiwari, and S. Nic Chormaic, Opt. Commun. 309 (2013) 313.

[19] R. Kumar, V. Gokhroo, K. Deasy, A. Maimaiti, M. C. Frawley, C. Phelan, and S. Nic Chormaic, New. J. Phys. 17 (2015) 013026.

[20] A. Stiebeiner, O. Rehband, R. Garcia-Fernandez, and A. Rauschenbeutel, Opt. Express 17 (2009) 21704.

[21] R. Yalla, Fam Le Kien, M. Morinaga, and K. Hakuta, Phys. Rev. Lett. 109 (2012) 063602.

[22] T. Schröder, M. Fujiwara, T. Noda, H.-Q. Zhao, O. Benson, and S. Takeuchi, Opt. Express 20 (2012) 10490.

[23] L. Liebermeister, F. Petersen, A. V. Münchow, D. Burchardt, J. Hermelbracht, T. Tashima, A. W. Schell, O. Benson, T. Meinhardt, A. Krueger, A. Stiebeiner, A. Rauschenbeutel, H. Weinfurter, and M. Weber, Appl. Phys. Lett. 104 (2014) 031101.

[24] G. Brambilla, G. S. Murugan, J. S. Wilkinson, and D. J. Richardson, Opt. Lett. 32 (2007) 3041.

[25] S. E. Skelton, M. Sergides, R. Patel, E. Karczewska, O. M. Maragó, and P. H. Jones, J. Quant. Spectrosc. Radiat. Transfer 113 (2012) 2512.

[26] Fam Le Kien and A. Rauschenbeutel, Phys. Rev. A 88 (2013) 063845.

[27] A. Maimaiti, Viet Giang Truong, M. Sergides, I. Gusachenko, and S. Nic Chormaic, Sci. Rep. 5 (2015) 09077.

[28] A. Maimaiti, D. Holzmann, Viet Giang Truong, H. Ritsch, and S. Nic Chormaic, Sci. Rep. 6 (2016) 30131.

[29] P. Lodahl, S. Mahmoodian, S. Stobbe, P. Schneeweiss, J. Volz, A. Rauschenbeutel, H. Pichler, and P. Zoller, Nature (London) 541 (2017) 473.

[30] Fam Le Kien and A. Rauschenbeutel, Phys. Rev. A 90 (2014) 023805.

[31] J. Petersen, J. Volz, and A. Rauschenbeutel, Science 346 (2014) 67.

[32] R. Mitsch, C. Sayrin, B. Albrecht, P. Schneeweiss, and A. Rauschenbeutel, Nature Commun. 5 (2014) 5713.

[33] Fam Le Kien and A. Rauschenbeutel, Phys. Rev. A 90 (2014) 063816.

[34] C. Sayrin, C. Junge, R. Mitsch, B. Albrecht, D. O’Shea, P. Schneeweiss, J. Volz, and A. Rauschenbeutel, Phys. Rev. X 5 (2015) 041036.

[35] K. Y. Bliokh, J. Dressel, and F. Nori, New J. Phys. 16 (2014) 093037.

[36] K. Y. Bliokh, A. Y. Bekshaev, and F. Nori, Nature Commun. 5 (2014) 3300.

[37] K. Y. Bliokh and F. Nori, Phys. Rep. 592 (2015) 1.

[38] A. V. Dooghin, N. D. Kundikova, V. S. Liberman, and B. Y. Zeldovich, Phys. Rev. A 45 (1992) 8204; V. S. Liberman and B. Y. Zeldovich, Phys. Rev. A 46 (1992) 5199; M. Y. Darsht, B. Y. Zeldovich, I. V. Kataevskaya, and N. D. Kundikova, JETP 80 (1995) 817 [Zh. Eksp. Theor. Phys. 107 (1995) 1464].

[39] K. Y. Bliokh, A. Aiello, and M. A. Alonso, in The Angular Momentum of Light, D. L. Andrews and M. Babiker, eds. (Cambridge University Press, 2012), p. 174.

[40] A. Aiello, P. Banzer, M. Neugebauer, and G. Leuchs, Nature Photon. 9 (2015) 789.

[41] K. Y. Bliokh, F. J. Rodriguez-Fortuño, F. Nori, and A. V. Zayats, Nature Photon. 9 (2015) 796. 
[42] Y. Tang and A. E. Cohen, Phys. Rev. Lett. 104 (2010) 163901.

[43] Y. Tang and A. E. Cohen, Science 332 (2011) 333.

[44] See, for example, D. Marcuse, Light Transmission Optics (Krieger, Malabar, FL, 1989); A. W. Snyder and J. D. Love, Optical Waveguide Theory (Chapman and Hall, New York, 1983); K. Okamoto, Fundamentals of Optical Waveguides (Elsevier, New York, 2006).

[45] Fam Le Kien, Th. Busch, Viet Giang Truong, and Síle Nic Chormaic, ArXiv: 1703.00109.

[46] G. Volpe and D. Petrov, Opt. Commun. 237 (2004) 89.

[47] A. Petcu-Colan, M. Frawley, and S. Nic Chormaic, J. Nonlinear Opt. Phys. Mat. 20 (2011) 293.

[48] M. C. Frawley, A. Petcu-Colan, V. G. Truong, and S. Nic Chormaic, Opt. Commun. 285 (2012) 4648.

[49] S. Ravets, J. E. Hoffman, L. A. Orozco, S. L. Rolston, G. Beadie, and F. K. Fatemi, Opt. Express 21 (2013) 18325.

[50] J. M. Ward, A. Maimaiti, V. H. Le, and S. Nic Chormaic, Rev. Sci. Instrum. 85 (2014) 111501.

[51] G. Nienhuis, Phys. Rev. A 93 (2016) 023840.

[52] D. Lipkin, J. Math. Phys. 5 (1964) 696.

[53] K. Y. Bliokh and F. Nori, Phys. Rev. A 83 (2011) 021803.

[54] M. M. Coles and D. L. Andrews, Phys. Rev. A 85 (2012) 063810.

[55] A. B. Harris, R. D. Kamien, and T. C. Lubensky, Rev. Mod. Phys. 71 (1999) 1745.

[56] R. P. Cameron, S. M. Barnett, and A. M. Yao, New J. Phys. 14 (2012) 053050.

[57] D. J. Candlin, Nuovo Cimento 37 (1965) 1390.

[58] J. L. Trueba and A. F. Ranada, Eur. J. Phys. 17 (1996) 141.

[59] G. N. Afanasiev and Y. P. Stepanovsky, Nuovo Cimento A 109 (1996) 271.

[60] T. G. Philbin, Phys. Rev. A 87 (2013) 043843. 